\title{
SYNTHESIS OF NEW PYRANE AND CYCLOHEXANONE DERIVATIVES ON THE BASE OF SOME KNOEVENAGEL ADDUCTS
}

\author{
F.N.Naghiyev \\ Baku State University \\ farid.orgchemist@gmail.com
}

Received 21.06.2019

Accepted 10.09.2019

\begin{abstract}
The formation of cyclohexanone and 3,4-dihydro-2H-pyran derivatives by using of Michael addition reaction between the 3-(4-tolyl)-, 3-(4-methoxyphenyl)-, 3-phenyl-, 3-pyridinyl-2-(thiophene-2-carbonyl)acrylonitriles or 3-(4-tolyl)-, 3-(thiophen-2-yl), and 2-(4-bromobenzoyl)-3-(4-fluorophenyl)acrylonitriles to the acetoasetanilid were observed. Structures of all synthesized compounds confirmed by nuclear magnetic resonance spectroscopy and X-Ray analysis.
\end{abstract}

Keywords: thiophene, acrylonitriles, acetoacetanilide, cyclohexanone, dihydropyran, nuclear magnetic resonance.

\section{doi.org/10.32737/0005-2531-2020-2-39-47}

Dihydropyrane, cyclohexanone derivatives have high biologically activity and widely extended in nature. In the literatures there are a lot of information on synthesis and various transformation of this type compounds [1-15]. Also, the Michael addition is the fundamental reaction of formation of $\mathrm{C}-\mathrm{C}$ bond in organic synthesis and we discussed it in early investigations [16-29].

In article [8] was presented Michael addition of acetoacetanilide to Knoevenagel adducts. So, it was estabilished that by Michael interaction of 2-(thiophene-2-carbonyl)-3-( $p$-to- lyl)-acrylonitrile and 3-(4-methoxyphenyl)-2(thiophene-2-carbonyl)acrylonitrile with acetoacetanilide in methanol at room temperature as dihydropyrane so cyclohexanone [8] derivatives were formed. Reaction schemes presented in below. As seen from the scheme, for obtaining of cyclohexanone derivatives, the methyleneactive compound (3) transferred to anion (4) by the action of base at first stage and at next stage obtained anion attacks to carbonyl group and occurs closing cycle by formation of cyclohexanone derivatives $(5 a, b)$.<smiles></smiles> 
Also, it was established, that for obtaining pyrane derivative by the same reaction, formed methyleneactive compound (or corresponding Michael adduct) (3) transferred to enol-form (6). Further, as result of interaction of electron pair of oxygen of hydroxyl to carbon of keton group taken place cycle formation by formation of dihydropyrane derivatives $(7 a, b)$.

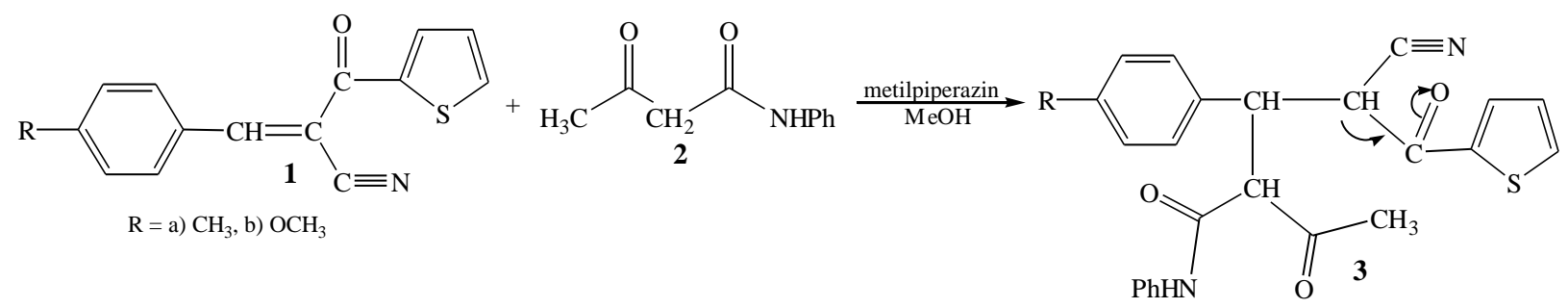<smiles>[R]c1ccc(C2C(C#N)=C(c3cccs3)OC(C)(O)C2C(=O)Nc2ccccc2)cc1</smiles>

By the Michael addition of 3-phenyl-2-(thiophene-2-carbonyl)acrylonitrile and 3-(pyridine4-yl)-2-(thiophene-2-carbonyl)acrylonitrile with acetoacetanilide at the same conditions (by the same mechanism) the dihydropyrane deriva- tives with high yield and cyclohexanone derivatives with low yield are synthesized. The X-Ray molecular structure of dihydropyrane derivative $(9 b)$ is given in below (Figure).<smiles>N#CC(=Cc1ccccc1)C(=O)c1cccs1</smiles><smiles>CC(=O)CC(=O)Nc1ccccc1</smiles>
$\frac{\text { metilpiperazin }}{\mathrm{MeOH}}$<smiles>[Y]OC1(C)OC(c2cccs2)=C(C#N)C(c2cc[Y]cc2)C1C(=O)Nc1ccccc1</smiles> 


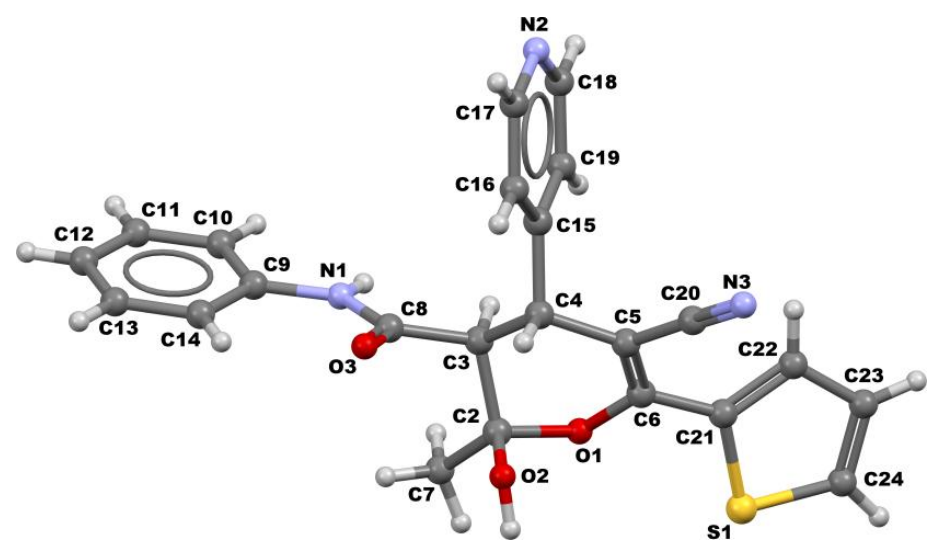

$\mathrm{X}$-Ray structure of 5-cyano-2-hydroxy-2-methyl-N-phenyl-4-(pyridin-4-yl)-6(thiophen-2-yl)-3,4-dihydro-2H-pyran-3-carboxamide (9b).

By the Michael addition of 2-(4-bromobenzoyl)-3-(4-fluorophenyl)acrylonitrile, 2-(4-bromobenzoyl)-3-(thiophene-2-yl)acrylonitrile and 2-(4-bromobenzoyl)-3-(p-tolyl)acrylonitrile with acetoacetanilide the similar products (12 a,b,c and $13 a, b, c)$ were synthesized.

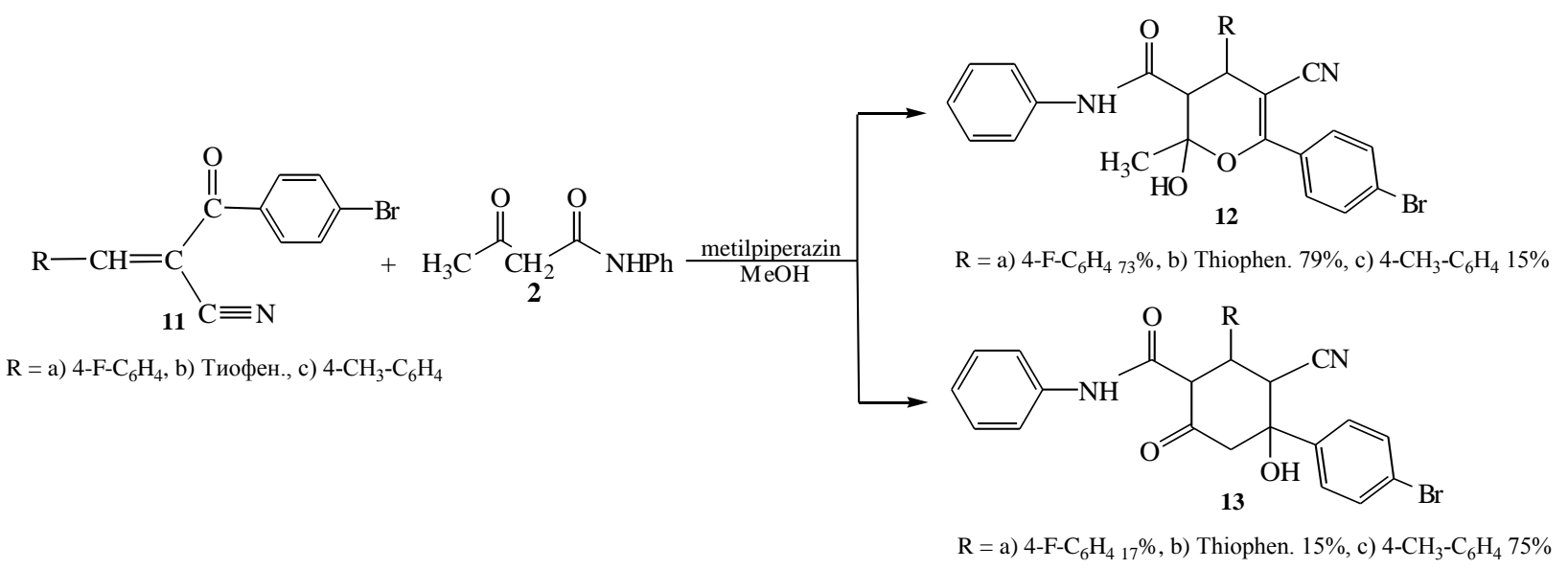

\section{Experimental part. General remarks}

All commercially available chemicals were obtained from Merck and Fluka (sigmaaldrich) companies and used without further purification. Melting points were measured on an Stuart SMP30 apparatus without correction. ${ }^{1} \mathrm{H}$, ${ }^{13} \mathrm{C}$ NMR spectra were recorded on BrukerAvance $300-\mathrm{MHz}$ spectrometer at 300 and 75 $\mathrm{MHz}$, respectively. X-Ray analyses were performed on Bruker APEX X-Ray equipment. Thin-layer chromatography (TLC) on commercial aluminum-backed plates of silica gel (60
F254) was used to monitor the progress of reactions.

\section{General experimental procedure}

3-Cyano-4-hydroxy-6-oxo-N-phenyl-4(thiophen-2-yl)-2-( $p$-tolyl)cyclohexane-1-carboxamide (5a). Mixture of 2-(thiophene-2carbonyl)-3-(p-tolyl)acrylonitrile $(2.9 \mathrm{mmol})$ and acetoacetanilide ( $3 \mathrm{mmol}$ ) dissolved in $35 \mathrm{ml}$ of methanol was stirrered 5-7 minutes and 2-3 drop methylpiperazine was added and stirring was continued. Reaction progress was tracked by TLC (EtOAc/n-hexane, 2:1). Reaction mixture was kept quietly for $24-48$ hours. By 
evaporating of solvent crystals were precipitated. Crystals were separated by filter paper and recrystalliized from ethanol $(95 \%)$ - water mixture. Yield $-0.89 \mathrm{~g}, 71.77 \% . T_{\text {melt. }}=210^{\circ} \mathrm{C}$.

${ }^{1} \mathrm{H}$ NMR (300 MHz, DMSO-d $d_{6}$ ), $\delta$, m.h.: $2.23\left(\mathrm{~s}, 3 \mathrm{H}, \mathrm{CH}_{3}\right) ; 2.79\left(\mathrm{~d}, 1 \mathrm{H}, \mathrm{CH},{ }^{3} J_{\mathrm{H}-\mathrm{H}}=\right.$ $14.1) ; 3.50\left(\mathrm{~d}, 1 \mathrm{H}, \mathrm{CH},{ }^{3} J_{\mathrm{H}-\mathrm{H}}=13.8\right) ; 4.01(\mathrm{~s}$, $1 \mathrm{H}, \mathrm{OH}) ; 4.06\left(\mathrm{~s}, 2 \mathrm{H}, \mathrm{CH}_{2}\right) ; 4.28(\mathrm{~d}, 1 \mathrm{H}, \mathrm{CH}$, $\left.{ }^{3} J_{\mathrm{H}-\mathrm{H}}=9.9\right) ; \quad 6.97-7.48 \quad(\mathrm{~m}, \quad 12 \mathrm{H}$, $\left.9 \mathrm{Ar}-\mathrm{H}+3 \mathrm{CH}_{\text {thioph. }}\right) ; 9.94$ (s, 1H, NH). ${ }^{13} \mathrm{C} \mathrm{NMR}$ spektr (DMSO-d $\left.)_{6}\right), \delta$, m.h.: $21.14\left(\mathrm{CH}_{3}-\mathrm{Ar}\right)$, $44.26(\mathrm{CH}), 47.40(\mathrm{CH}), 54.07\left(\mathrm{CH}_{2}\right), 62.64$ $(\mathrm{CH}), \quad 75.29 \quad\left(\mathrm{C}_{\text {quat }}\right), \quad 119.02 \quad(\mathrm{CN}), \quad 119.49$ (2 $\left.\mathrm{CH}_{\text {arom }}\right), 123.87\left(\mathrm{CH}_{\text {thioph. }}\right), 124.45\left(\mathrm{CH}_{\text {arom }}\right)$, 125.71 ( $\left.\mathrm{CH}_{\text {thioph. }}\right), 127.63\left(\mathrm{CH}_{\text {thioph. }}\right), 128.75$ $\left(2 \mathrm{CH}_{\text {arom }}\right), 129.14\left(2 \mathrm{CH}_{\text {arom }}\right), 129.54\left(2 \mathrm{CH}_{\text {arom }}\right)$, $137.06\left(\mathrm{C}_{\text {ar. }}\right), 137.17\left(\mathrm{C}_{\mathrm{ar}}\right), 139.14\left(\mathrm{C}_{\mathrm{ar} .}\right), 150.57$ $\left(\mathrm{C}_{\text {thioph. }}\right), 165.85(\mathrm{O}=\underline{\mathrm{C}}-\mathrm{NH}), 203.12(\mathrm{C}=\mathrm{O})$.

Found, \%: $69.71 \mathrm{C}, 5.07 \mathrm{H}, 6.56 \mathrm{~N}$. $\mathrm{C}_{25} \mathrm{H}_{22} \mathrm{~N}_{2} \mathrm{O}_{3} \mathrm{~S}$. Calculated, \%: $69.77 \mathrm{C}, 5.12 \mathrm{H}$, $6.51 \mathrm{~N}$.

3-Cyano-4-hydroxy-2-(4-methoxyphenyl)-6-oxo-N-phenyl-4-(thiophen-2-yl)-cyclohexane-1-carboxamide $(\mathbf{5} b)$ was synthesized in the same conditions by using of 3-(4-methoxyphenyl)-2-(thiophene-2-carbonyl)acrylonitrile (yield $1.1 \mathrm{~g}, 85.27 \%$ ). $T_{\text {melt. }}=200^{\circ} \mathrm{C}$.

${ }^{1} \mathrm{H}$ NMR (300 MHz, DMSO- $\left.d_{6}\right), \delta$, m.h.: $2.77\left(\mathrm{~d}, 1 \mathrm{H}, \mathrm{CH},{ }^{3} J_{\mathrm{H}-\mathrm{H}}=14.1\right) ; 3.48(\mathrm{~d}, 1 \mathrm{H}, \mathrm{CH}$, $\left.{ }^{3} J_{\mathrm{H}-\mathrm{H}}=14\right) ; 3.56(\mathrm{~s}, 1 \mathrm{H}, \mathrm{OH}) ; 3.69(\mathrm{~s}, 3 \mathrm{H}$, $\left.\mathrm{CH}_{3} \mathrm{O}\right) ; 4.00(\mathrm{~s}, 1 \mathrm{H}, \mathrm{OH}) ; 4.03\left(\mathrm{~s}, 2 \mathrm{H}, \mathrm{CH}_{2}\right)$; $4.27\left(\mathrm{~d}, 1 \mathrm{H}, \mathrm{CH},{ }^{3} J_{\mathrm{H}-\mathrm{H}}=9.6\right) ; 6.89-7.47(\mathrm{~m}, 12 \mathrm{H}$, 9Ar-H+3 $\left.\mathrm{CH}_{\text {thioph. }}\right) ; 9.93$ (s, $\left.1 \mathrm{H}, \mathrm{NH}\right) .{ }^{13} \mathrm{C} \mathrm{NMR}$ spektr (DMSO- $\left.d_{6}\right), \delta$, m.h.: $43.88(\mathrm{CH}), 47.51$ $(\mathrm{CH}), 54.06\left(\mathrm{CH}_{2}\right), 55.37\left(\mathrm{CH}_{3} \mathrm{O}\right), 62.79(\mathrm{CH})$, $75.22\left(\mathrm{C}_{\text {quat. }}\right), 114.23\left(2 \mathrm{CH}_{\text {arom }}\right), 119.07(\mathrm{CN})$, $119.52\left(2 \mathrm{CH}_{\text {arom }}\right), 123.87\left(\mathrm{CH}_{\text {thioph. }}\right), 124.42$ $\left(\mathrm{CH}_{\text {arom }}\right), 125.70\left(\mathrm{CH}_{\text {thioph. }}\right), 127.62\left(\mathrm{CH}_{\text {thioph. }}\right)$, $129.14\left(2 \mathrm{CH}_{\text {arom }}\right), 129.98\left(2 \mathrm{CH}_{\text {arom }}\right), 131.96$ $\left(\mathrm{C}_{\text {ar. }}\right), 139.13\left(\mathrm{C}_{\text {ar. }}\right), 150.59$ ( $\left.\mathrm{C}_{\text {thioph }}\right), 158.90$ $\left(\mathrm{O}^{-} \underline{\mathrm{C}}_{\text {ar. }}\right), 165.90(\mathrm{O}=\underline{\mathrm{C}}-\mathrm{NH}), 203.14(\mathrm{C}=\mathrm{O})$.

Found, \%: $67.31 \mathrm{C}, 4.99 \mathrm{H}, 6.23 \mathrm{~N}$. $\mathrm{C}_{25} \mathrm{H}_{22} \mathrm{~N}_{2} \mathrm{O}_{4} \mathrm{~S}$. Calculated, \%: $67.26 \mathrm{C}, 4.93 \mathrm{H}$, $6.28 \mathrm{~N}$.

5-Cyano-2-hydroxy-2-methyl-N-phenyl-6-(thiophen-2-yl)-4-(p-tolyl)-3,4-dihydro2H-pyran-3-carboxamide (7a) was synthesized in the same conditions by using of 2- (thiophene-2-carbonyl)-3-(p-tolyl)acrylonitrile (yield $0.25 \mathrm{~g}, 20.16 \%$ ). $T_{\text {melt. }}=188^{\circ} \mathrm{C}$.

${ }^{1} \mathrm{H}$ NMR (300 MHz, DMSO- $\left.d_{6}\right), \delta$, m.h.: $1.76\left(\mathrm{~s}, 3 \mathrm{H}, \mathrm{CH}_{3}\right) ; 2.26\left(\mathrm{~s}, 3 \mathrm{H}, \mathrm{CH}_{3}\right) ; 3.03(\mathrm{~d}$, $\left.1 \mathrm{H}, \mathrm{CH},{ }^{3} J_{\mathrm{H}-\mathrm{H}}=11.5\right) ; 3.46(\mathrm{~s}, 1 \mathrm{H}, \mathrm{OH}) ; 4.39$ $\left(\mathrm{d}, 1 \mathrm{H}, \mathrm{CH},{ }^{3} J_{\mathrm{H}-\mathrm{H}}=11.5\right) ; 6.94-7.51(\mathrm{~m}, 12 \mathrm{H}$, $\left.9 \mathrm{Ar}-\mathrm{H}+3 \mathrm{CH}_{\text {thioph. }}\right) ; 9.84(\mathrm{~s}, 1 \mathrm{H}, \mathrm{NH}) .{ }^{13} \mathrm{C}$ NMR spektr (DMSO-d6), $\delta$, m.h.: $21.00\left(\mathrm{CH}_{3}\right)$, $26.26\left(\mathrm{CH}_{3}\right), 39.67(\mathrm{CH}), 54.96(\mathrm{CH}), 84.14$ $\left(=\mathrm{C}_{\text {quat. }}\right), 95.28\left(\mathrm{O}-\underline{\mathrm{C}}_{\text {quat. }}\right), 119.62\left(2 \mathrm{CH}_{\text {arom }}\right)$, $120.12 \quad(\mathrm{CN}), \quad 124.11 \quad\left(\mathrm{CH}_{\text {arom }}\right), \quad 124.67$ (2 $\left.\mathrm{CH}_{\text {arom }}\right), 127.72\left(\mathrm{CH}_{\text {thioph. }}\right), 128.78\left(\mathrm{CH}_{\text {thioph. }}\right)$, $129.39\left(2 \mathrm{CH}_{\text {arom }}\right), 129.86\left(2 \mathrm{CH}_{\text {arom }}\right), 129.54$ $\left(\mathrm{CH}_{\text {thioph. }}\right), 138.31 \quad\left(\mathrm{CH}_{3}-\mathrm{C}_{\text {ar. }}\right), 139.23\left(\mathrm{C}_{\text {ar. }}\right)$, $\begin{array}{llll}140.16 & \left(\mathrm{C}_{\text {ar. }}\right), \quad 141.85 \quad\left(\mathrm{C}_{\text {thioph }}\right), & 165.98\end{array}$ $\left(\mathrm{O}-\underline{\mathrm{C}}_{\text {quat. }}=\right), 166.64(\underline{\mathrm{CONH}})$.

Found, \%: $69.82 \mathrm{C} ; 5.18 \mathrm{H} ; 6.46 \mathrm{~N}$. $\mathrm{C}_{25} \mathrm{H}_{22} \mathrm{~N}_{2} \mathrm{O}_{3} \mathrm{~S}$. Calculated, \%: $69.77 \mathrm{C} ; 5.12 \mathrm{H}$; $6.51 \mathrm{~N}$.

5-Cyano-2-hydroxy-4-(4-methoxyphenyl)-2-methyl-N-phenyl-6-(thiophen-2-yl)-3,4dihydro-2H-pyran-3-carboxamide (7b) was synthesized in the same conditions by using of 3-(4-methoxyphenyl)-2-(thiophene-2-carbonyl)acrylonitrile(yield $\quad 0.16 \quad \mathrm{~g}, \quad 12.40 \%$ ). Tmelt $=181^{\circ} \mathrm{C}$.

${ }^{1} \mathrm{H}$ NMR (300 MHz, DMSO- $\left.d_{6}\right), \delta$, m.h.: $1.79\left(\mathrm{~s}, 3 \mathrm{H}, \mathrm{CH}_{3}\right) ; 3.05\left(\mathrm{~d}, 1 \mathrm{H}, \mathrm{CH},{ }^{3} J_{\mathrm{H}-\mathrm{H}}=\right.$ $11.3) ; 3.42(\mathrm{~s}, 1 \mathrm{H}, \mathrm{OH}) ; 4.41\left(\mathrm{~d}, 1 \mathrm{H}, \mathrm{CH},{ }^{3} \mathrm{~J}_{\mathrm{H}-\mathrm{H}}=\right.$ 11.3); 6.98-7.59 (m, 12H, 9Ar-H + 3CH $\left.\mathrm{CH}_{\text {thioph. }}\right)$; $9.87(\mathrm{~s}, 1 \mathrm{H}, \mathrm{NH}) .{ }^{13} \mathrm{C}$ NMR spektr (DMSO-d 6 ), $\delta$, m.h.: $26.32\left(\mathrm{CH}_{3}\right), 39.81(\mathrm{CH}), 55.05(\mathrm{CH})$, $55.93\left(\mathrm{OCH}_{3}\right), 84.85\left(=\mathrm{C}_{\text {quat. }}\right), 96.02\left(\mathrm{O}-\mathrm{C}_{\text {quat. }}\right)$, $115.77\left(2 \mathrm{CH}_{\text {arom }}\right), 121.58\left(2 \mathrm{CH}_{\text {arom }}\right), 119.86$ $(\mathrm{CN}), \quad 124.92 \quad\left(\mathrm{CH}_{\text {arom }}\right), \quad 127.80 \quad\left(\mathrm{CH}_{\text {thioph. }}\right)$, $128.82\left(\mathrm{CH}_{\text {thioph. }}\right), 129.71 \quad\left(\mathrm{CH}_{\text {thioph. }}\right), 130.05$ $\left(2 \mathrm{CH}_{\text {arom }}\right), 131.17\left(2 \mathrm{CH}_{\text {arom }}\right), 137.34 \quad\left(\mathrm{C}_{\mathrm{ar}}\right)$, $140.08\left(\mathrm{C}_{\mathrm{ar}}\right), 142.26\left(\mathrm{C}_{\text {thioph. }}\right), 159.71\left(\mathrm{O}-\underline{\mathrm{C}}_{\mathrm{ar}}\right)$, $165.62\left(\mathrm{O}-\underline{\mathrm{C}}_{\text {quat. }}=\right), 166.68(\underline{\mathrm{CONH}})$.

Found, \%: $67.32 \mathrm{C}, 4.98 \mathrm{H}, 6.24 \mathrm{~N}$. $\mathrm{C}_{25} \mathrm{H}_{22} \mathrm{~N}_{2} \mathrm{O}_{4} \mathrm{~S}$. Calculated, \%: $67.26 \mathrm{C}, 4.93 \mathrm{H}$, $6.28 \mathrm{~N}$.

5-Cyano-2-hydroxy-2-methyl-N,4-diphenyl-6-(thiophen-2-yl)-3,4-dihydro-2H-pyran-3-carboxamide (9a). Mixture of 3-phenyl2-(thiophene-2-carbonyl)acrylonitrile $(2.9 \mathrm{mmol})$ and acetoacetanilide ( $3 \mathrm{mmol}$ ) dissolved in 35 $\mathrm{ml}$ of methanol was stirrered 5-7 $\mathrm{min}$ and 2-3 
drop methylpiperazine was added and stirring was continued. Reaction progress was tracked by TLC (EtOAc/n-hexane, 2:1). Reaction mixture was kept quietly for $24-48$ hours. By evaporating of solvent crystals were precipitated. Crystals were separated by filter paper and recrystalliized from ethanol $(95 \%)$ - water mixture. Yield $-0.9 \mathrm{~g}, 75 \% . T_{\text {melt. }}=186^{\circ} \mathrm{C}$.

${ }^{1} \mathrm{H}$ NMR (300 MHz, DMSO- $\left.d_{6}\right), \delta$, m.h.: $1.72\left(\mathrm{~s}, 3 \mathrm{H}, \mathrm{CH}_{3}\right) ; 3.07\left(\mathrm{~d}, 1 \mathrm{H}, \mathrm{CH},{ }^{3} \mathrm{~J}_{\mathrm{H}-\mathrm{H}}=\right.$ 11.7); 3.57 (s, 1H, OH); $4.38\left(\mathrm{~d}, 1 \mathrm{H}, \mathrm{CH},{ }^{3} \mathrm{~J}_{\mathrm{H}-\mathrm{H}}\right.$ $=11.7) ; \quad 7.00-7.89(\mathrm{~m}, \quad 13 \mathrm{H}, \quad 10 \mathrm{Ar}-\mathrm{H}+$ $\left.3 \mathrm{CH}_{\text {thioph. }}\right) ; 9.86(\mathrm{~s}, 1 \mathrm{H}, \mathrm{NH}) .{ }^{13} \mathrm{C}$ NMR spektr (DMSO- $\left.d_{6}\right), \delta$, m.h.: $26.38\left(\mathrm{CH}_{3}\right), 40.62(\mathrm{CH})$, $55.87(\mathrm{CH}), 86.25$ (= $\left.\mathrm{C}_{\text {quat. }}\right), 99.73\left(\mathrm{O}-\mathrm{C}_{\text {quat. }}\right)$, $119.58(\mathrm{CN}), \quad 119.66 \quad\left(2 \mathrm{CH}_{\text {arom }}\right), \quad 124.15$ $\left(\mathrm{CH}_{\text {arom }}\right), 128.03 \quad\left(\mathrm{CH}_{\text {arom }}\right), \quad 128.47\left(\mathrm{CH}_{\text {thioph. }}\right)$, $128.60\left(\mathrm{CH}_{\text {arom }}\right), \quad 128.87\left(2 \mathrm{CH}_{\text {arom }}\right), 129.05$ $\left(3 \mathrm{CH}_{\text {arom }}\right), 129.78\left(\mathrm{CH}_{\text {thioph. }}\right), 130.63\left(\mathrm{CH}_{\text {thioph. }}\right)$, 135.99 ( $\left.\mathrm{C}_{\text {ar. }}\right), 138.87$ ( $\left.\mathrm{C}_{\text {thioph. }}\right), 140.12\left(\mathrm{C}_{\mathrm{ar} .}\right)$, $166.77\left(\mathrm{O}-\underline{\mathrm{C}}_{\text {quat. }}=\right), 167.63(\mathrm{O}=\underline{\mathrm{C}}-\mathrm{NH})$.

Found, \%: $69.28 \mathrm{C}, 4.75 \mathrm{H}, 6.78 \mathrm{~N}$. $\mathrm{C}_{24} \mathrm{H}_{20} \mathrm{~N}_{2} \mathrm{O}_{3} \mathrm{~S}$. Calculated, \%: $69.23 \mathrm{C}, 4.81 \mathrm{H}$, $6.73 \mathrm{~N}$.

5-Cyano-2-hydroxy-2-methyl-N-phenyl4-(pyridin-4-yl)-6-(thiophen-2-yl)-3,4-dihydro2H-pyran-3-carboxamide (9b). Mixture 4-pyridinecarboxaldehyde or $(2.9 \mathrm{mmol})$ and 2-thenoylacetonitrile $(3 \mathrm{mmol})$ dissolved in $50 \mathrm{ml}$ of ethanol-water (4:1) was stirrered 5 minutes, reaction mixture was kept quietly for 36 hours. Then, added acetoacetanilide $(3 \mathrm{mmol})$ to reaction mixture, stirring for $5 \mathrm{~min}$ and 2-3 drop methylpiperazine was added. Reaction mixture was kept quietly for 24 hours. Reaction progress was tracked by TLC (EtOAc/n-hexane, 2:1). By evaporating of solvent crystals were precipitated. Crystals were separated by filter paper and recrystalliized from ethanol (95\%) water mixture. Yield $0.97 \mathrm{~g}, 79.51 \%$. $T_{\text {melt. }}=$ $165^{\circ} \mathrm{C}$.

${ }^{1} \mathrm{H}$ NMR (300 MHz, DMSO- $\left.d_{6}\right), \delta$, m.h.: $1.70\left(\mathrm{~s}, 3 \mathrm{H}, \mathrm{CH}_{3}\right) ; 3.05\left(\mathrm{~d}, 1 \mathrm{H}, \mathrm{CH},{ }^{3} \mathrm{~J}_{\mathrm{H}-\mathrm{H}}=\right.$ $11.4) ; 3.88(\mathrm{~s}, 1 \mathrm{H}, \mathrm{OH}) ; 4.39\left(\mathrm{~d}, 1 \mathrm{H}, \mathrm{CH},{ }^{3} \mathrm{~J}_{\mathrm{H}-\mathrm{H}}\right.$ $=11.4) ; 7.03-8.56\left(\mathrm{~m}, 12 \mathrm{H}, 9 \mathrm{Ar}-\mathrm{H}+3 \mathrm{CH}_{\text {thioph }}\right)$; $9.88(\mathrm{~s}, 1 \mathrm{H}, \mathrm{NH}) .{ }^{13} \mathrm{C}$ NMR spektr (DMSO-d 6 ), $\delta$, m.h.: $26.31\left(\mathrm{CH}_{3}\right), 40.21(\mathrm{CH}), 55.04(\mathrm{CH})$, $84.45\left(=\mathrm{C}_{\text {quat. }}\right), 99.72\left(\mathrm{O}-\underline{\mathrm{C}}_{\text {quat }}\right), 119.71(\mathrm{CN})$, $120.02\left(2 \mathrm{CH}_{\text {arom }}\right), \quad 124.29 \quad\left(\mathrm{CH}_{\text {arom }}\right), \quad 124.44$ $\left(\mathrm{CH}_{\text {arom }}\right), 128.52\left(\mathrm{CH}_{\text {thioph. }}\right), 129.21 \quad\left(3 \mathrm{CH}_{\text {arom }}\right)$, $130.11\left(\mathrm{CH}_{\text {thioph. }}\right), 130.97\left(\mathrm{CH}_{\text {thioph. }}\right), 135.67\left(\mathrm{C}_{\text {ar. }}\right)$, $138.68\left(\mathrm{C}_{\text {thioph }}\right), 149.17\left(\mathrm{C}_{\text {ar. }}\right), 150.38\left(2 \mathrm{CH}_{\text {arom }}\right)$, $166.68\left(\mathrm{O}-\underline{\mathrm{C}}_{\text {quat. }}=\right), 167.23(\mathrm{O}=\underline{\mathrm{C}}-\mathrm{NH})$.

Found, \%: $66.14 \mathrm{C} ; 4.51 \mathrm{H} ; 10.13 \mathrm{~N}$. $\mathrm{C}_{23} \mathrm{H}_{19} \mathrm{~N}_{3} \mathrm{O}_{3} \mathrm{~S}$. Calculated, \%: $66.19 \mathrm{C} ; 4.56 \mathrm{H}$; $10.07 \mathrm{~N}$.

\section{3-Cyano-4-hydroxy-6-oxo-N,2-diphe-} nyl-4-(thiophen-2-yl)cyclohexane-1-carboxamide (10a) was synthesized in the same conditions by using of 3-phenyl-2-(thiophene-2carbonyl)acrylonitrile(yield $0.22 \mathrm{~g}, 18.33 \%$ ). $T_{\text {melt }}=206^{0} \mathrm{C}$.

${ }^{1} \mathrm{H}$ NMR (300 MHz, DMSO- $\left.d_{6}\right), \delta$, m.h.: ${ }^{1} \mathrm{H}$ NMR spektr (DMSO-d6), $\delta$, m.h.: 2.72 (d, $\left.1 \mathrm{H}, \mathrm{CH},{ }^{3} J_{\mathrm{H}-\mathrm{H}}=14\right) ; 3.47\left(\mathrm{~d}, 1 \mathrm{H}, \mathrm{CH},{ }^{3} J_{\mathrm{H}-\mathrm{H}}=\right.$ 13.5); 3.97 (s, 1H, OH); $4.01\left(\mathrm{~s}, 2 \mathrm{H}, \mathrm{CH}_{2}\right) ; 4.25$ $\left(\mathrm{d}, 1 \mathrm{H}, \mathrm{CH},{ }^{3} \mathrm{~J}_{\mathrm{H}-\mathrm{H}}=9.4\right) ; 7.05-7.85(\mathrm{~m}, 13 \mathrm{H}$, $\left.10 \mathrm{Ar}-\mathrm{H}+3 \mathrm{CH}_{\text {thioph. }}\right) ; 9.91 \quad(\mathrm{~s}, \quad 1 \mathrm{H}, \quad \mathrm{NH}) .{ }^{13} \mathrm{C}$ NMR spektr (DMSO-d6), $\delta$, m.h.: $44.46(\mathrm{CH})$, $47.65(\mathrm{CH}), 54.18\left(\mathrm{CH}_{2}\right), 62.83(\mathrm{CH}), 75.28$ $\left(\mathrm{O}-\underline{C}_{\text {quat }}\right), 116.27 \quad\left(2 \mathrm{CH}_{\text {arom. }}\right), \quad 119.10 \quad(\mathrm{CN})$, $123.11 \quad\left(\mathrm{CH}_{\text {thioph. }}\right), \quad 124.55 \quad\left(\mathrm{CH}_{\text {arom. }}\right), \quad 125.77$ $\left(\mathrm{CH}_{\text {thioph. }}\right), 127.68\left(\mathrm{CH}_{\text {arom. }}\right), 127.82\left(\mathrm{CH}_{\text {thioph. }}\right)$, $128.91\left(2 \mathrm{CH}_{\text {arom. }}\right), 129.33\left(2 \mathrm{CH}_{\text {arom }}\right), 129.79$ $\left(2 \mathrm{CH}_{\text {arom}}\right), 137.68\left(\mathrm{C}_{\mathrm{ar} .}\right), 139.30\left(\mathrm{C}_{\mathrm{ar} .}\right), 150.52$ $\left(\mathrm{C}_{\text {thioph. }}\right), 165.72(\mathrm{O}=\underline{\mathrm{C}}-\mathrm{NH}), 203.08(\underline{\mathrm{C}}=\mathrm{O})$.

Found, \%: $69.17 \mathrm{C} ; 4.86 \mathrm{H} ; 6.68 \mathrm{~N}$. $\mathrm{C}_{24} \mathrm{H}_{20} \mathrm{~N}_{2} \mathrm{O}_{3} \mathrm{~S}$. Calculated, \%: $69.23 \mathrm{C} ; 4.81 \mathrm{H}$; $6.73 \mathrm{~N}$.

3-Cyano-4-hydroxy-6-oxo-N-phenyl-2(pyridin-4-yl)-4-(thiophen-2-yl)cyclohexane1-carboxamide (10b) was synthesized in the same conditions by using of 3-(pyridin-4-yl)-2(thiophene-2-carbonyl)acrylonitrile(yield $0.19 \mathrm{~g}$, $15.70 \%) . T_{\text {melt. }}=194^{0} \mathrm{C}$.

${ }^{1} \mathrm{H}$ NMR (300 MHz, DMSO- $\left.d_{6}\right), \delta$, m.h.: ${ }^{1} \mathrm{H}$ NMR spektr (DMSO-d $), \delta$, m.h.: $2.76(\mathrm{~d}$, $\left.1 \mathrm{H}, \mathrm{CH},{ }^{3} J_{\mathrm{H}-\mathrm{H}}=14\right) ; 3.50\left(\mathrm{~d}, 1 \mathrm{H}, \mathrm{CH},{ }^{3} J_{\mathrm{H}-\mathrm{H}}=\right.$ 13.5); 3.99 (s, 1H, OH); 4.05 (s, 2H, $\left.\mathrm{CH}_{2}\right) ; 4.29$ $\left(\mathrm{d}, 1 \mathrm{H}, \mathrm{CH},{ }^{3} J_{\mathrm{H}-\mathrm{H}}=9.4\right) ; 7.07-8.62(\mathrm{~m}, 12 \mathrm{H}$, $\left.9 \mathrm{Ar}-\mathrm{H}+3 \mathrm{CH}_{\text {thioph. }}\right) ; 9.96(\mathrm{~s}, 1 \mathrm{H}, \mathrm{NH}) .{ }^{13} \mathrm{C} \mathrm{NMR}$ spektr (DMSO-d $), \delta$, m.h.: $43.79(\mathrm{CH}), 47.48$ $(\mathrm{CH}), 53.99\left(\mathrm{CH}_{2}\right), 62.76(\mathrm{CH}), 75.32\left(\mathrm{O}-\mathrm{C}_{\text {quat. }}\right)$, $\begin{array}{llll}118.24 & \left(2 \mathrm{CH}_{\text {arom. }}\right), \quad 119.15 \quad(\mathrm{CN}), \quad 123.24\end{array}$ $\left(\mathrm{CH}_{\text {thioph. }}\right), 124.59\left(\mathrm{CH}_{\text {arom. }}\right), 125.81\left(\mathrm{CH}_{\text {thioph. }}\right)$, $126.14\left(2 \mathrm{CH}_{\text {arom. }}\right), \quad 127.87\left(\mathrm{CH}_{\text {thioph }}\right), 129.86$ 
$\left(2 \mathrm{CH}_{\text {arom}}\right), 139.36\left(\mathrm{C}_{\mathrm{ar}}\right), 149.31\left(\mathrm{C}_{\text {ar. }}\right), 149.78$ $\left(2 \mathrm{CH}_{\text {arom. }}\right), 150.56\left(\mathrm{C}_{\text {thioph }}\right), 165.88(\mathrm{O}=\underline{\mathrm{C}}-\mathrm{NH})$, $203.12(\mathrm{C}=\mathrm{O})$.

Found, \%: $66.24 \mathrm{C} ; 4.62 \mathrm{H} ; 10.02 \mathrm{~N}$. $\mathrm{C}_{23} \mathrm{H}_{19} \mathrm{~N}_{3} \mathrm{O}_{3} \mathrm{~S}$. Calculated, \%: $66.19 \mathrm{C} ; 4.56 \mathrm{H}$; $10.07 \mathrm{~N}$.

\section{6-(4-Bromophenyl)-5-cyano-4-(4-fluo-} rophenyl)-2-hydroxy-2-methyl-N-phenyl-3,4dihydro-2H-pyran-3-carboxamide (12a). Mixture of 2-(4-bromobenzoyl)-3-(4-fluorophenyl)acrylonitrile $(2.9 \mathrm{mmol})$ and acetoacetanilide (3 mmol) dissolved in $35 \mathrm{ml}$ of methanol was stirrered 5-7 $\mathrm{min}$ and 2-3 drop methylpiperazine was added and stirring was continued. Reaction progress was tracked by TLC (EtOAc/n-hexane, 2:1). Reaction mixture was kept quietly for 24-48 hours. By evaporating of solvent crystals were precipitated. Crystals were separated by filter paper and recrystalliized from ethanol $(95 \%)$ - water mixture. Yield 1.08 $\mathrm{g}, 73.47 \% . T_{\text {melt }}=135^{\circ} \mathrm{C}$.

${ }^{1} \mathrm{H}$ NMR (300 MHz, DMSO- $\left.d_{6}\right), \delta$, m.h.: $1.71\left(\mathrm{~s}, 3 \mathrm{H}, \mathrm{CH}_{3}\right) ; 3.02\left(\mathrm{~d}, 1 \mathrm{H}, \mathrm{CH},{ }^{3} J_{\mathrm{H}-\mathrm{H}}=\right.$ 11.7); $3.52(\mathrm{~s}, 1 \mathrm{H}, \mathrm{OH}) ; 4.35\left(\mathrm{~d}, 1 \mathrm{H}, \mathrm{CH},{ }^{3} J_{\mathrm{H}-\mathrm{H}}\right.$ $=11.7) ; 7.00-7.97(\mathrm{~m}, 13 \mathrm{H}, 13 \mathrm{Ar}-\mathrm{H}) ; 9.85(\mathrm{~s}$, $1 \mathrm{H}, \mathrm{NH}) .{ }^{13} \mathrm{C}$ NMR spektr (DMSO-d 6 ), $\delta$, m.h.: $26.37\left(\mathrm{CH}_{3}\right), 39.88(\mathrm{CH}), 55.94(\mathrm{CH}), 89.19$ ( $\left.=\mathrm{C}_{\text {quat. }}\right), 99.65$ (O-C $\left.\mathrm{C}_{\text {quat. }}\right), 115.79-116.07\left(\mathrm{CH}_{\text {arom }}\right)$, $119.70(\mathrm{CN}), 120.00\left(2 \mathrm{CH}_{\text {arom }}\right), 124.19-124.87$ $\left(\mathrm{CH}_{\text {arom }}\right), 129.17\left(3 \mathrm{CH}_{\text {arom }}\right), 130.70\left(3 \mathrm{CH}_{\text {arom }}\right)$, 131.08 ( $\left.\mathrm{CH}_{\text {arom }}\right), \quad 131.99\left(2 \mathrm{CH}_{\text {arom }}\right), \quad 132.85$ $\left(\mathrm{Br}-\mathrm{C}_{\mathrm{ar}}\right), 136.13-136.16 \quad\left(\mathrm{C}_{\mathrm{ar} .}\right), 138.81 \quad\left(2 \mathrm{C}_{\mathrm{ar}}\right)$, $160.32-167.71 \quad\left(\mathrm{~F}-\underline{\mathrm{C}}_{\mathrm{ar}}.\right), \quad 161.51 \quad\left(=\underline{\mathrm{C}}_{\text {quat. }} . \mathrm{O}\right)$, $166.75(\mathrm{HN}-\underline{\mathrm{C}}=\mathrm{O})$.

Found, \%: $61.60 \mathrm{C} ; 3.99 \mathrm{H} ; 5.47 \mathrm{~N}$. $\mathrm{C}_{26} \mathrm{H}_{20} \mathrm{~N}_{2} \mathrm{O}_{3} \mathrm{FBr}$. Calculated, \%: $61.54 \mathrm{C} ; 3.94 \mathrm{H}$; $5.52 \mathrm{~N}$.

6-(4-Bromophenyl)-5-cyano-2-hydroxy-2methyl-N-phenyl-4-(thiophen-2-yl)-3,4-dihydro2H-pyran-3-carboxamide (12b) was synthesized in the same conditions by using of 2-(4-bromobenzoyl)-3-(thiophen-2-yl)acrylonitrile (yield $0.95 \mathrm{~g}, 79.17 \%) . T_{\text {melt }}=117^{\circ} \mathrm{C}$.

${ }^{1} \mathrm{H}$ NMR (300 MHz, DMSO- $d 6$ ), $\delta$, m.h.: $1.70\left(\mathrm{~s}, 3 \mathrm{H}, \mathrm{CH}_{3}\right) ; 3.06\left(\mathrm{~d}, 1 \mathrm{H}, \mathrm{CH},{ }^{3} J_{\mathrm{H}-\mathrm{H}}=\right.$ $11.7) ; 3.58(\mathrm{~s}, 1 \mathrm{H}, \mathrm{OH}) ; 4.63\left(\mathrm{~d}, 1 \mathrm{H}, \mathrm{CH},{ }^{3} \mathrm{~J}_{\mathrm{H}-\mathrm{H}}\right.$ $=11.7) ; \quad 6.99-7.88 \quad(\mathrm{~m}, \quad 12 \mathrm{H}, \quad 9 \mathrm{Ar}-\mathrm{H}+$ $\left.3 \mathrm{CH}_{\text {thiophen. }}\right) ; 10.01$ (s, $\left.1 \mathrm{H}, \mathrm{NH}\right) .{ }^{13} \mathrm{C} \mathrm{NMR}$ spektr $\left(\mathrm{DMSO}-d_{6}\right), \delta$, m.h.: $26.27\left(\mathrm{CH}_{3}\right), 35.95$ $(\mathrm{CH}), 56.42(\mathrm{CH}), 89.56\left(=\mathrm{C}_{\text {quat. }}\right), 99.85(\mathrm{O}-$ $\left.\mathrm{C}_{\text {quat. }}\right), 119.69(\mathrm{CN}), 119.90\left(2 \mathrm{CH}_{\text {arom }}\right), 124.24$ ( $\left.\mathrm{Br}-\mathrm{C}_{\text {ar }}\right), 126.06\left(\mathrm{CH}_{\text {thiophen. }}\right), 127.34\left(\mathrm{CH}_{\text {thiophen. }}\right)$, $127.51\left(\mathrm{CH}_{\text {thiophen. }}\right), 129.23\left(2 \mathrm{CH}_{\text {arom }}\right), 130.68$ $\left(3 \mathrm{CH}_{\text {arom }}\right), 132.09\left(2 \mathrm{CH}_{\text {arom }}\right), 132.14\left(\mathrm{C}_{\text {ar. }}\right), 138.86$ $\left(\mathrm{C}_{\text {thioph. }}\right), 142.63\left(\mathrm{C}_{\text {ar. }}\right), 161.02\left(=\underline{\mathrm{C}}_{\text {quat }} . \mathrm{O}\right), 166.56$ $(\mathrm{HN}-\underline{\mathrm{C}}=\mathrm{O})$.

Found, \%: $69.35 \mathrm{C} ; 4.64 \mathrm{H} ; 6.70 \mathrm{~N}$. $\mathrm{C}_{24} \mathrm{H}_{19} \mathrm{~N}_{2} \mathrm{O}_{3} \mathrm{~S}$. Calculated, \%: $69.40 \mathrm{C} ; 4.58 \mathrm{H}$; $6.75 \mathrm{~N}$.

6-(4-Bromophenyl)-5-cyano-2-hydroxy2-methyl-N-phenyl-4-(p-tolyl)-3,4-dihydro-2Hpyran-3-carboxamide (12c) was synthesized in the same conditions by using of 2-(4-bromobenzoyl)-3-(p-tolyl)acrylonitrile (yield $0.21 \mathrm{~g}$, $14.48 \%) \cdot T_{\text {melt }}=207^{\circ} \mathrm{C}$.

${ }^{1} \mathrm{H}$ NMR (300 MHz, DMSO-d6), $\delta$, m.h.: $1.74\left(\mathrm{~s}, 3 \mathrm{H}, \mathrm{CH}_{3}\right) ; 2.30$ (s, 3H, $\left.\mathrm{Ar}-\underline{\mathrm{CH}}_{3}\right) ; 3.03$ (d, $\left.1 \mathrm{H}, \mathrm{CH},{ }^{3} J_{\mathrm{H}-\mathrm{H}}=11.7\right) ; 3.57(\mathrm{~s}, 1 \mathrm{H}, \mathrm{OH}) ; 4.45$ $\left(\mathrm{d}, 1 \mathrm{H}, \mathrm{CH},{ }^{3} J_{\mathrm{H}-\mathrm{H}}=11.7\right) ; 7.05-7.92(\mathrm{~m}, 13 \mathrm{H}$, 13Ar-H); $9.90(\mathrm{~s}, 1 \mathrm{H}, \mathrm{NH}) .{ }^{13} \mathrm{C} \mathrm{NMR}$ spektr (DMSO- $\left.d_{6}\right), \delta$, m.h.: $21.14\left(\mathrm{CH}_{3}-\mathrm{Ar}\right), 26.32$ $\left(\mathrm{CH}_{3}\right), 40.47(\mathrm{CH}), 55.57(\mathrm{CH}), 86.36\left(=\mathrm{C}_{\text {quat. }}\right)$, 99.33 (O-C quat. $), 119.61(\mathrm{CN}), 119.73\left(2 \mathrm{CH}_{\text {arom }}\right)$, $124.18\left(\mathrm{CH}_{\text {arom }}\right), 125.06\left(2 \mathrm{CH}_{\text {arom }}\right), 125.13(\mathrm{Br}-$ $\left.\underline{\mathrm{C}}_{\text {ar. }}\right), 128.49\left(2 \mathrm{CH}_{\text {arom }}\right), 130.51\left(2 \mathrm{CH}_{\text {arom }}\right), 130.87$ $\left(2 \mathrm{CH}_{\text {arom }}\right), \quad 133.15\left(2 \mathrm{CH}_{\text {arom }}\right), \quad 135.28 \quad\left(\mathrm{C}_{\text {ar. }}\right)$, $139.78\left(\mathrm{C}_{\mathrm{ar}}\right), 140.65\left(\mathrm{C}_{\mathrm{ar}}\right), 141.27\left(\mathrm{C}_{\mathrm{ar}}\right), 161.35$ $\left(\mathrm{O}-\underline{\mathrm{C}}_{\text {quat. }}=\right), 166.64(\mathrm{O}=\underline{\mathrm{C}}-\mathrm{NH})$.

Found, \%: $64.46 \mathrm{C} ; 4.63 \mathrm{H} ; 5.52 \mathrm{~N}$. $\mathrm{C}_{27} \mathrm{H}_{23} \mathrm{~N}_{2} \mathrm{O}_{3} \mathrm{Br}$. Calculated, \%: $64.41 \mathrm{C} ; 4.57 \mathrm{H}$; $5.57 \mathrm{~N}$.

4-(4-Bromophenyl)-3-cyano-2-(4-fluorophenyl)-4-hydroxy-6-oxo-N-phenylcyclohexane-1-carboxamide (13a) was synthesized in the same conditions by using of 2-(4-bromobenzoyl)-3-(4-fluorophenyl)acrylonitrile (yield $0.28 \mathrm{~g}, 17.69 \%) . T_{\mathrm{melt}}=164^{0} \mathrm{C}$.

${ }^{1} \mathrm{H}$ NMR (300 MHz, DMSO- $\left.d_{6}\right), \delta$, m.h.: $2.84\left(\mathrm{~d}, 1 \mathrm{H}, \mathrm{CH},{ }^{3} J_{\mathrm{H}-\mathrm{H}}=14.1\right) ; 3.58(\mathrm{~d}, 1 \mathrm{H}$, $\left.\mathrm{CH},{ }^{3} J_{\mathrm{H}-\mathrm{H}}=13.8\right) ; 4.08(\mathrm{~s}, 1 \mathrm{H}, \mathrm{OH}) ; 4.11(\mathrm{~s}$, $\left.2 \mathrm{H}, \mathrm{CH}_{2}\right) ; 4.35\left(\mathrm{~d}, 1 \mathrm{H}, \mathrm{CH},{ }^{3} J_{\mathrm{H}-\mathrm{H}}=9.9\right) ; 7.03-$ $7.62\left(\mathrm{~m}, 12 \mathrm{H}, 9 \mathrm{Ar}-\mathrm{H}+3 \mathrm{CH}_{\text {thioph. }}\right) ; 9.97(\mathrm{~s}, 1 \mathrm{H}$, $\mathrm{NH}) .{ }^{13} \mathrm{C}$ NMR spektr (DMSO- $d_{6}$ ), $\delta$, m.h.: $45.18(\mathrm{CH}), 47.89(\mathrm{CH}), 54.21\left(\mathrm{CH}_{2}\right), 62.72$ (CH), 75.31 (O- $\left.\underline{C}_{\text {quat }}\right), 115.92-116.20\left(\mathrm{CH}_{\text {arom }}\right)$, $119.12(\mathrm{CN}), 119.34\left(2 \mathrm{CH}_{\text {arom }}\right), 123.21-123.49$ 
$\left(\mathrm{CH}_{\text {arom }}\right), 125.56\left(\mathrm{CH}_{\text {arom }}\right), 129.23\left(3 \mathrm{CH}_{\text {arom }}\right)$, $130.45\left(2 \mathrm{CH}_{\text {arom }}\right), \quad 130.98 \quad\left(\mathrm{CH}_{\text {arom }}\right), \quad 131.82$ $\left(2 \mathrm{CH}_{\mathrm{arom}}\right), 132.85\left(\mathrm{Br}-\mathrm{C}_{\mathrm{ar}}\right), 135.92-135.95\left(\mathrm{C}_{\mathrm{ar}}\right)$, $138.22\left(\mathrm{C}_{\mathrm{ar} .}\right), \quad 138.26\left(\mathrm{C}_{\mathrm{ar}}\right), \quad 160.46-166.96$ $\left(\mathrm{C}_{\mathrm{ar} .}\right), 165.93(\mathrm{O}=\underline{\mathrm{C}}-\mathrm{NH}), 203.05(\underline{\mathrm{C}}=\mathrm{O})$.

Found, \%: $61.49 \mathrm{C} ; 3.88 \mathrm{H} ; 5.57 \mathrm{~N}$. $\mathrm{C}_{26} \mathrm{H}_{20} \mathrm{~N}_{2} \mathrm{O}_{3} \mathrm{FBr}$. Calculated, \%: $61.54 \mathrm{C} ; 3.94 \mathrm{H}$; $5.52 \mathrm{~N}$

\section{4-(4-Bromophenyl)-3-cyano-4-hydroxy- 6-oxo-N-phenyl-2-(thiophen-2-yl)cyclohexane- 1-carboxamide (13b) was synthesized in the} same conditions by using of 2-(4-bromobenzoyl)-3-(thiophen-2-yl)acrylonitrile (yield $0.18 \mathrm{~g}, 15 \%) . T_{\text {melt }}=143^{\circ} \mathrm{C}$.

${ }^{1} \mathrm{H}$ NMR (300 MHz, DMSO- $d 6$ ), $\delta$, m.h.: $2.74\left(\mathrm{~d}, 1 \mathrm{H}, \mathrm{CH},{ }^{3} J_{\mathrm{H}-\mathrm{H}}=14.2\right) ; 3.55(\mathrm{~d}, 1 \mathrm{H}$, $\left.\mathrm{CH},{ }^{3} J_{\mathrm{H}-\mathrm{H}}=13.8\right) ; 3.96(\mathrm{~s}, 1 \mathrm{H}, \mathrm{OH}) ; 4.09(\mathrm{~s}$, $\left.2 \mathrm{H}, \mathrm{CH}_{2}\right) ; 4.24\left(\mathrm{~d}, 1 \mathrm{H}, \mathrm{CH},{ }^{3} J_{\mathrm{H}-\mathrm{H}}=9.9\right) ; 7.02-$ $7.54\left(\mathrm{~m}, 12 \mathrm{H}, 9 \mathrm{Ar}-\mathrm{H}+3 \mathrm{CH}_{\text {thioph. }}\right) ; 9.91(\mathrm{~s}, 1 \mathrm{H}$, $\mathrm{NH}) .{ }^{13} \mathrm{C}$ NMR spektr (DMSO-d6), $\delta$, m.h.:44.18 (CH), $47.36(\mathrm{CH}), 54.11\left(\mathrm{CH}_{2}\right)$, $62.57(\mathrm{CH}), 75.42\left(\mathrm{O}-\underline{\mathrm{C}}_{\text {quat. }}\right), 119.08(\mathrm{CN})$, $119.38\left(2 \mathrm{CH}_{\text {arom }}\right), 123.07$ (Br- $\left.\underline{\mathrm{C}}_{\text {ar. }}\right), 125.14$ $\left(\mathrm{CH}_{\text {arom. }}\right), 125.39\left(\mathrm{CH}_{\text {thioph. }}\right), 128.08\left(\mathrm{CH}_{\text {thioph. }}\right)$, $128.48\left(2 \mathrm{CH}_{\text {arom. }}\right), 128.62 \quad\left(\mathrm{CH}_{\text {thioph. }}\right), 129.44$ $\left(2 \mathrm{CH}_{\text {arom }}\right), 131.28\left(2 \mathrm{CH}_{\text {arom }}\right), 136.67 \quad\left(\mathrm{C}_{\text {ar. }}\right)$, $139.14 \quad\left(\mathrm{C}_{\text {ar. }}\right), \quad 149.98 \quad\left(\mathrm{C}_{\text {thioph. }}\right), \quad 165.69$ $(\mathrm{O}=\underline{\mathrm{C}}-\mathrm{NH}), 203.02(\underline{\mathrm{C}}=\mathrm{O})$.

Found, \%: $69.45 \mathrm{C} ; 4.53 \mathrm{H} ; 6.81 \mathrm{~N}$. $\mathrm{C}_{24} \mathrm{H}_{19} \mathrm{~N}_{2} \mathrm{O}_{3} \mathrm{~S}$. Calculated, \%: $69.40 \mathrm{C} ; 4.58 \mathrm{H}$; $6.75 \mathrm{~N}$.

4-(4-Bromophenyl)-3-cyano-4-hydroxy6-oxo-N-phenyl-2-(p-tolyl)cyclohexane-1-carboxamide (13c) was synthesized in the same conditions by using of 2-(4-bromobenzoyl)-3(p-tolyl)acrylonitrile (yield $1.10 \mathrm{~g}, 75.86 \%$ ). $T_{\text {melt }}=238^{0} \mathrm{C}$.

${ }^{1} \mathrm{H}$ NMR (300 MHz, DMSO-d6), $\delta$, m.h.: $2.28\left(\mathrm{~s}, 3 \mathrm{H}, \mathrm{CH}_{3}\right) ; 2.85\left(\mathrm{~d}, 1 \mathrm{H}, \mathrm{CH},{ }^{3} J_{\mathrm{H}-\mathrm{H}}=\right.$ 13.7); $3.54(\mathrm{~s}, 1 \mathrm{H}, \mathrm{OH}) ; 3.67\left(\mathrm{~d}, 1 \mathrm{H}, \mathrm{CH},{ }^{3} \mathrm{~J}_{\mathrm{H}-\mathrm{H}}\right.$ $=13.7) ; 4.12\left(\mathrm{~s}, 2 \mathrm{H}, \mathrm{CH}_{2}\right) ; 4.30(\mathrm{~d}, 1 \mathrm{H}, \mathrm{CH}$, $\left.{ }^{3} J_{\mathrm{H}-\mathrm{H}}=10.0\right) ; 7.14-7.65(\mathrm{~m}, 13 \mathrm{H}, 13 \mathrm{Ar}-\mathrm{H}) ; 9.97$ $(\mathrm{s}, 1 \mathrm{H}, \mathrm{NH}),{ }^{13} \mathrm{C}$ NMR spektr (DMSO- $\left.d_{6}\right), \delta$, m.h.: $21.18\left(\underline{\mathrm{CH}}_{3}-\mathrm{Ar}\right), 44.36(\mathrm{CH}), 47.51(\mathrm{CH})$, $54.19\left(\mathrm{CH}_{2}\right), 62.71(\mathrm{CH}), 76.04\left(\mathrm{O}-\mathrm{C}_{\text {quat. }}\right), 119.15$ $(\mathrm{CN}), 119.44\left(2 \mathrm{CH}_{\text {arom }}\right), 123.14\left(\mathrm{Br}-\mathrm{C}_{\text {ar }}\right), 125.37$ $\left(\mathrm{CH}_{\text {arom. }}\right), 128.45\left(2 \mathrm{CH}_{\text {arom }}\right), 129.17\left(2 \mathrm{CH}_{\text {arom }}\right)$, $129.26\left(2 \mathrm{CH}_{\text {arom. }}\right), 130.23\left(2 \mathrm{CH}_{\text {arom. }}\right), 131.97$ $\left(2 \mathrm{CH}_{\text {arom }}\right), 137.46\left(\mathrm{C}_{\text {ar. }}\right), 137.77\left(\mathrm{C}_{\text {ar. }}\right), 139.70$ $\left(\mathrm{C}_{\mathrm{ar} .}\right), 142.11\left(\mathrm{C}_{\mathrm{ar}}\right), 165.92(\mathrm{O}=\underline{\mathrm{C}}-\mathrm{NH}), 202.92$ $(\mathrm{C}=\mathrm{O})$.

Found, \%: $64.35 \mathrm{C} ; 4.52 \mathrm{H} ; 5.62 \mathrm{~N}$. $\mathrm{C}_{27} \mathrm{H}_{23} \mathrm{~N}_{2} \mathrm{O}_{3}$ Br. Calculated, \%: $64.41 \mathrm{C} ; 4.57 \mathrm{H}$; $5.57 \mathrm{~N}$.

\section{References}

1. Niu Zh., He X., Shang Y. The efficient enantioselective synthesis of dihydropyrans via organocatalytic Michael addition reactions. Tetrahedron: Asymmetry. 2014. V. 25. Iss. 10-11. P. 796-801.

2. Wu B., Gao X., Yan Zh., Hung W.-X., Zhou Y.-G. Enantioselective synthesis of functionalized 2amino-4H-chromenes via the o-quinone methides generated from 2-(1-tosylalkyl)phenols. Tetrahedron Letters. 2015. V. 56. Iss. 29. P. 4334-4338.

3. Duan C., Ye L., Xu W., Li X., Chen F., Zhao Zh., Li X. Organocatalytic cascade 1,6-conjugate addition/annulation/tautomerization of functionalized para-quinone methides: Access to chiral 2-amino4-aryl-4H-chromenes. Chinese Chemical Letters. 2018. V. 29. Iss. 8. P. 1273-1276.

4. Feng J., Fu X., Chen Zh., Lin L., Liu X., and Feng $X$. Efficient Enantioselective Synthesis of Dihydropyrans Using a Chiral N,N'-Dioxide as Organocatalyst. Org. Lett. 2013. V. 15. Iss. 11. P. 2640-2643.

5. Desimoni G., Faita G., and Quadrelli P. Forty Years after "Heterodiene Syntheses with $\alpha, \beta$-Unsaturated Carbonyl Compounds": Enantioselective Syntheses of 3,4-Dihydropyran Derivatives. Chem. Rev. 2018. V. 118. Iss. 4. P. 2080-2248.

6. Feng J., Yuan X., Luo W., Lin L., Liu X., Feng X. Chiral N,N'-Dioxide-Organocatalyzed Regio-, Diastereo- and Enantioselective Michael Addition-Alkylation Reaction. Chem. A Europ. J. 2016. V. 22. Iss. 44. P. 15650-15653.

7. Xie J., Xing W.-L., Sha F., Wu X.-Y. Enantioselective Cascade Reaction of $\alpha$-Cyano Ketones and Isatylidene Malononitriles: Asymmetric Construction of Spiro[4H-pyran-oxindoles]. Europ. J. Organic Chem. 2016. No 23. P. 3983-3992.

8. Naghiyev F.N., Maharramov A.M., Asgarova A.R., Rahimova A.G., Akhundova M.A., Mamedov I.G. Investigation of reaction of various thiophene basedknoevenagel adducts with acetoacetanilide. Chemical Problems. 2018. No 3. P. 337-342.

9. Li Ch., Cai L.-Zh., Liu X.-D., Zhu Sh.-Zh., Xing Ch.H., Lu L. Highly efficient synthesis of tetrasubstituted 2,3-dihydropyrans by three-component 'one-pot' reaction. Tetrahedron Letters. 2016. V. 57. Iss. 20. P. 2171-2174.

10. Pałasz A. Synthesis of 3,4-dihydro-2H-pyrans by hetero-Diels-Alder reactions of functionalized alpha,beta-unsaturated carbonyl compounds with $\mathrm{N}$ - 
vinyl-2-oxazolidinone. Org. Biomol. Chem. 2005. V. 3. Iss. 17. P. 3207-3212.

11. Kendre D.B., Toche R.B., Jachak M.N. Michael addition of dimedone with $\alpha, \beta$-unsaturated ketones: Synthesis of quinoline and chromene derivatives. Journal of Heterocyclic Compounds. 2008. V. 45. Iss. 3. P. 667-671.

12. Liu W., Zhou J., Zheng Ch., Chen X., Xiao H., Yang Y., Guo Y., Zhao G. Tandem cross-RauhutCurrier/cyclization reactions of activated alkenes to give densely functionalized 3,4-dihydropyrans. Tetrahedron. 2011. V. 67. Iss. 10. P. 1768-1773.

13. Zhang Q., Fang T., Tong X. Facile synthesis of highly functionalized six-membered heterocycles via $\mathrm{PPh}_{3}$-catalyzed [4+2] annulations of activated terminal alkynes and hetero-dienes: scope, mechanism, and application.Tetrahedron. 2010. V. 66. Iss. 40. P. 8095-8100.

14. Ni H., Yao W., Waheed A., Ullah N., and Lu Y. Enantioselective [4+2]-Annulation of Oxadienes and Allenones Catalyzed by an Amino Acid Derived Phosphine: Synthesis of Functionalized Dihydropyrans. Org. Lett.2016. V. 18. Iss. 9. P. 2138-2141.

15. Hu Zh.-P., Wang W.-J., Yin X.-G., Zhang X.-J., Yan M. Enantioselective synthesis of 2-amino-4H-pyrans via the organocatalytic cascade reaction of malononitrile and $\alpha$-substituted chalcones. Tetrahedron: Asymmetry. 2012. V. 23. Iss. 6-7. P. 461-467.

16. Maharramov A.M, Sadikhova N.D., Mammadov I.G., Allakhverdiev M.A. Alkylation of B-Dicarbonyl Compounds as a Method for the Production of Functionally Substituted Dihydrofurans. Chem. Heterocyc. Comp. 2009. V. 45. No 4. P. 400-404.

17. Mamedov I.G, Eichhoff U, Maharramov A.M, Bayramov M.R, Mamedova Y.V. Molecular Dynamics of Alkenylphenol Derivatives in Solution as Studied by NMR Spectroscopy. Appl. Magn. Reson. 2010. V. 38. Iss. 3. P. 257-269.

18. Mamedov I.G, Bayramov M.R, Mamedova Y.V, Maharramov A.M. Molecular dynamics of cis-1(2-hydroxy-5-methylphenyl)ethanoneoxime and $\mathrm{N}$-(2-hydroxy-4-methylphenyl)acetamide in solution studied by NMR spectroscopy. Magn. Reson. Chem. 2010. V. 48. Iss. 9. P. 671-677.

19. Mamedov I.G, Eichhoff U, Maharramov A.M, Bayramov M.R, Mamedova Y.V. Molecular dynamics of (Z)-1-(2-hydroxy-5-methyl-3-nitrophenyl)ethanoneoxime and (E)-2-hydroxy-5-methylacetophenone thiosemicarbazone in solution studied by NMR spectroscopy. Cent. Eur. J. Chem. 2012. V. 10. Iss. 1. P. 241-247.

20. Mamedov I.G, Bayramov M.R, Mamedova Y.V. Maharramov A.M. Molecular dynamics of (E)-6- acetyl-3-(2-hydroxy-5-methylphenyl)-5-styrylcyclohex-2-en-1-one and (E)-6-ethylcarboxylate-3-(2hydroxy-5-methylphenyl)-5-styryl cyclohex-2-en1 -one in a solution studied by NMR spectroscopy. Magn. Reson. Chem. 2013. V. 51. Iss. 9. P. 600604.

21. Mamedov I.G, Bayramov M.R, Mamedova Y.V, Maharramov A.M. New synthesis on the basis 2allyloxy chalcone and NMR studies its some derivatives. Magn. Reson. Chem. 2015. V. 53. Iss. 2. P. 147-153.

22. Mamedov I.G, Bayramov M.R, Salamova A.E, Maharramov A.M. NMR studies of some 4-hydroxy-2methylacetophenone thiosemicarbazones in solutions. Ind. Jour. Chem. 2015. 54 B. P. 1518-1527.

23. Mamedov I.G, Abbasoglu R, Bayramov M.R, Maharramov A.M. Synthesis of a new 1,2,3,4,5-pentasubstituted cyclohexanol and determining its stereochemistry by NMR spectroscopy and quantumchemical calculations. Magn. Reson. Chem. 2016. V. 54. Iss. 4. P. 315-319.

24. Mamedov I.G, Mamedova Y.V, Khrustalev V.N, Bayramov M.R, Maharramov A.M. Dependence of biological activities of some chalcone derivatives from the molecular structure. Ind. Jour. Chem. 2017. 56 B. P. 192-196.

25. Mamedov I.G, Farzaliyeva A.E, Mamedova Y.V, Hasanova N.N, Bayramov M.R, Maharramov A.M. Antibacterial; Antifungal; Anticancer; Acetophenone; NMR spectroscopy. İnd. Jour. Chem. 2018. 57 B. P. 1310-1314.

26. Maharramov A.M., Mamedova Y.V., Bayramov M.R., Mamedov I.G. Chalcone Derivatives As Corrosion Inhibitors for Mild Steel in Brine-Kerosene Solution. Russ. Jour. Phys. Chem. 2018. 92. P. 2154-2158.

27. Mamedov İ.G, Khrustalev V.N, Dorovatovskii P.V, Naghiev F.N, Maharramov A.M. Efficient synthesis of new tricyclic pyrano[3,2-c]pyridine derivatives. Mendeleev Communications. 2019. V. 29. Iss. 2. P. 232-233.

28. Naghiyev F.N, Mamedov İ.G, Khrustalev V.N, Shikhaliev N.G, MaharramovA.M. A new direction in the alkylation of 5-acetyl-2-amino-6methyl-4-phenyl-4H-pyran-3-carbonitrile with active methylene reagents. Journal of the Chinese Chemical Society. 2019. V. 66. Iss. 3. P. 253-256.

29. Maharramov A.M, Naghiyev F.N, Mamedova G.Z, Asadov Kh.A. Synthesis of Spiroindolines on the Basis of Isatylidene Malononitrile. Russian Journal of Organic Chemistry. 2018. V. 54. Iss. 11. P. 1731-1734. 


\section{BəZI KNOEVENAGEL ADDUKTLARI OSASINDA YENI PIRAN Və TSIKKLOHEKSANON TÖRӘMӘLӘRININN SINTEZI}

\section{F.N.Nağıyev}

3-(4-Tolil)-, 3-(4-metoksifenil)-, 3-fenil-, 3-piridinil-2-(tiofen-2-karbonil)akrilonitrillərin, eləcə də 3-(4-Tolil)-, 3-(thiophen-2-yl) və 2-(4-brombenzoil)-3-(4-flüorfenil)akrilonitrilin asetoasetanilid ilə Mixael birləşmə reaksiyasiyasından həm əvəzlənmiș tsikloheksanon, həm də 3,4-dihidro-2H-piran törəmələri alınmıșdır. Sintez edilmiş birləșmələrin quruluşları nüvə maqnit rezonansı spektroskopiyası və X-Ray analizinin köməyi ilə təsdiq edilmişdir.

Açar sözlor: tiofen, akrilonitrillar, asetoasetanilid, tsikloheksanon,dihidropiran, nüvə maqnit rezonansı spektroskopiyas1.

\section{СИНТЕЗ НОВЫХ ПРОИЗВОДНЫХ ПИРАНА И ЦИКЛОГЕКСАНОНА НА ОСНОВЕ НЕКОТОРЫХ АДДУКТОВ КНОВЕНАГЕЛЯ}

\section{Ф.Н.Нагиев}

Присоединением по Михаэлю ацетоацетанилида к 3-(4-толил)-, 3-(4-метоксифенил)-, 3-фенил-, 3-пиридинил-2(тиофен-2-карбонил)акрилонитрилам или 3-(4-толил)-, 3-(тиофен-2-ил) и 2-(4-бромбензоил)-3-(4-фторфенил)акрилонитрилам получены производные циклогексанона и 3,4-дигидро-2Н-пирана. Структуры всех синтезированных соединений подтверждены данными спектроскопией ядерно-магнитного резонанса и рентгенографии.

Ключевые слова: тиофен, акрилонитрилы, ацетоачетанилид, ичклогексанон, дигидропиран, спектроскопия ядерно-магнитного резонанса. 